\title{
Risk Factors for Incarceration in Patients with Primary Abdominal Wall and Incisional Hernias: A Prospective Study in 4472 Patients
}

\author{
Dimitri Sneiders ${ }^{1,6} \cdot$ Yagmur Yurtkap $^{1} \cdot$ Leonard F. Kroese $^{2} \cdot$ Gert-Jan Kleinrensink $^{3}$ • \\ Johan F. Lange ${ }^{1,4} \cdot$ Jean-François Gillion $^{5}$. The Hernia-Club Members
}

Published online: 12 April 2019

(C) The Author(s) 2019

\begin{abstract}
Background Incarceration of primary and incisional hernias often results in emergency surgery. The objective of this study was to evaluate the relation of defect size and location with incarceration. Secondary objectives comprised identification of additional patient factors associated with an incarcerated hernia.

Methods A registry-based prospective study was performed of all consecutive patients undergoing hernia surgery between September 2011 and February 2016. Multivariate logistic regression was performed to identify risk factors for incarceration.

Results In total, 83 (3.5\%) of 2352 primary hernias and 79 (3.7\%) of 2120 incisional hernias had a non-reducible incarceration. For primary hernias, a defect width of $3-4 \mathrm{~cm}$ compared to defects of $0-1 \mathrm{~cm}$ was significantly associated with an incarcerated hernia (OR 2.85, 95\% CI 1.57-5.18, $p=0.0006)$. For incisional hernias, a defect width of 3-4 cm compared to defects of $0-2 \mathrm{~cm}$ was significantly associated with an incarceration (OR $2.14,95 \% \mathrm{CI}$ $1.07-4.31, p=0.0324)$. For primary hernias, defects in the peri- and infra-umbilical region portrayed a significantly increased odds for incarceration as compared to supra-umbilical defects (OR 1.98, 95\% CI 1.02-3.85, $p=0.043$ ). Additionally, in primary hernias age, BMI, and constipation were associated with incarceration. In incisional hernias age, BMI, female sex, diabetes mellitus and ASA classification were associated with incarceration.

Conclusion For primary and incisional hernias, mainly defects of 3-4 cm were associated with incarceration. For primary hernias, mainly defects located in the peri- and infra-umbilical region were associated with incarceration. Based on patient and hernia characteristics, patients with increased odds for incarceration may be selected and these patients may benefit from elective surgical treatment.
\end{abstract}

Dimitri Sneiders and Yagmur Yurtkap have contributed equally to this study and both authors should be considered as first author.

Collaborating members are listed in the Acknowledgements.

Registration Number Commission Nationale de l'Informatique et des Libertés' (CNIL Registration Number: 1993959v0).

Electronic supplementary material The online version of this article (https://doi.org/10.1007/s00268-019-04989-x) contains supplementary material, which is available to authorized users.

Dimitri Sneiders

d.sneiders@erasmusmc.nl
1 Department of Surgery, Erasmus University Medical Center, Rotterdam, The Netherlands

2 Department of Surgery, Reinier de Graaf Gasthuis, Delft, The Netherlands

3 Department of Neuroscience and Anatomy, Erasmus University Medical Center, Rotterdam, The Netherlands

4 Department of Surgery, IJsselland Ziekenhuis, Capelle aan den IJssel, The Netherlands

5 Unité de Chirurgie Viscérale et Digestive, Hôpital Privé d'Antony, Antony, France

6 PO Box 2040, 3000 CA Rotterdam, The Netherlands 


$\begin{array}{ll}\text { Abbreviations } \\ \text { AAA } & \text { Abdominal aortic aneurysm } \\ \text { ASA } & \text { American Society of Anesthesiologists score } \\ \text { BMI } & \text { Body mass index } \\ \text { OR } & \text { Odds ratio } \\ \text { ROC } & \text { Receiver operator characteristic }\end{array}$

\section{Introduction}

Abdominal wall hernias may result in pain, discomfort and aesthetic dissatisfaction and remain an important surgical challenge [1]. Moreover, hernias may be associated with significant morbidity and in rare cases mortality due to incarceration of bowel or abdominal contents such as fat or omentum [2-4]. Incarceration of the bowel is an absolute indication for emergency surgery. Previous research has shown prevalence rates of $4-15 \%$ of abdominal wall hernias resulting in emergency surgery. Emergency surgery is associated with severely compromised outcomes and increased mortality as compared to elective hernia repair $[2,3,5,6]$.

Risk of incarceration may be increased due to factors increasing intra-abdominal pressure. Obesity, ascites, chronic cough, and constipation are factors that all have been reported to increase intra-abdominal pressure [4, 7-9]. Hernia characteristics such as defect location and defect size may be associated with incarceration as well. Smaller defects are often thought to be at increased risk for incarceration; however, the evidence supporting this theory is limited. In fact, a previous study found no evidence for an increased incarceration risk in defects below $2 \mathrm{~cm}$ and another recent study found no association at all between defect size and hernia incarceration [4, 6].

The primary objective of this prospective study was to evaluate the relation of defect size and location with incarceration in primary and incisional hernias. Secondary objectives comprised identification of additional patient factors associated with an incarcerated hernia.

\section{Methods}

This prospective study was conducted within the French Hernia-Club registry. The Hernia-Club registry is approved by the French 'Commission Nationale de l'Informatique et des Libertés' (CNIL registration number: 1993959v0). Since this study is registry based and guaranties completely anonymized data, additional participant and institutional review board approval were not required according to the Dutch and French national standards. This study was conducted according to the STROBE (Strengthening the Reporting of Observational studies in Epidemiology) recommendations for observational studies [10].

\section{Study design}

A registry-based prospective study was performed including all adult patients in the French Hernia-Club registry that underwent hernia repair surgery, for primary or incisional hernias, between September 1, 2012 and February 29, 2016. Patients with incarcerated hernias were compared to patients without an incarcerated hernia. The present study differentiates between two types of incarceration as determined during surgery. The first type constitutes of a non-reducible protrusion of abdominal contents (e.g. fat, omentum, or bowel) through the abdominal wall defect. A hernia was considered non-reducible if reintegration of contents was only possible after adhesiolysis or enlarging of the defect. The second type constitutes of incarcerated hernias that could be easily manually reduced without the need for adhesiolysis or enlargement of the defect. Only the first type of incarceration, i.e. non-reducible incarceration was considered as endpoint for the present analysis. Cases without information on incarceration were considered as non-informative and subsequently excluded from further analysis.

\section{Hernia-club registry}

The Hernia-Club registry is a prospective and anonymized online database of all surgical procedures for primary and incisional hernias. The registry contains data of abdominal wall surgery performed in academic and non-academic centres by 47 surgeons. Each participating specialist must accept and sign the Charter of Quality. This states that: 'all input must be registered in a consecutive, unselected and exhaustive manner and in real time.' Data from screening, pre-, peri- and postoperative periods are collected in real time through online forms by the operating surgeon. A total of 164 parameters are collected. To ensure high-quality data, participants consent to random peer review of the original medical charts. Within a follow-up period of 2 years, outcomes are collected by the surgeon and further checked by an independent research associate. In case of discrepancies in collected data, the medical records are checked. The collected parameters in this database are fully compatible with the European Hernia Society (EHS) classification of primary and incisional abdominal wall hernias and the European Registry of Abdominal Wall Hernias (EuraHS) international online platform [11, 12]. 


\section{Data collection}

For the present study, predefined patient baseline characteristics and hernia characteristics were extracted from the Hernia-Club registry. Baseline characteristics of interest comprised age, body mass index (BMI), sex, current smoking habits, diabetes mellitus, corticosteroid use, radiotherapy, chemotherapy, history of abdominal aortic aneurysm (AAA), collagen disorder, anticoagulant use, history of abdominal hernia (inguinal, primary or incisional), family history of abdominal hernia, American Society of Anesthesiologists (ASA) score, and primary surgery (none, gastro-intestinal, gynaecologic, or other). Factors of interest related to increased intra-abdominal pressure comprised ascites, chronic cough, constipation (i.e. frequent episodes of no defecation lasting for more than 3 days), and heavy lifting (i.e. patients who have to carry more than $10 \mathrm{~kg}$ multiple times a day). Hernia characteristics comprised hernia type (primary or incisional), defect location (supra-umbilical, (peri)-umbilical, infra-umbilical, or lateral), defect width, recurrent hernia, and previous surgery with mesh. Data on defect width was measured either by physical examination alone or by physical and radiological examination. Defect width was only available in whole centimetres. Defect width was categorized in 4 categories for primary hernias $(1 \mathrm{~cm}$, $2 \mathrm{~cm}, 3-4 \mathrm{~cm}, \geq 5 \mathrm{~cm})$ and for incisional hernias $(1-2 \mathrm{~cm}$, $3-4 \mathrm{~cm}, 5-10 \mathrm{~cm},>10 \mathrm{~cm})$.

\section{Statistical analysis}

Statistical analysis was performed with RStudio (Version 1.0.153_(C) 2009-2017 RStudio, Inc.) [13]. Data on primary and incisional hernias were analysed separately. Categorical variables are presented as absolute numbers and percentages. Continuous variables are presented as means with corresponding standard deviations (SD). Missing data is presented in absolute numbers and percentage for each variable of interest in the Supplement. Normality of continuous variables was assessed with Levene's test for the equality of variances and graphically in histograms. Differences between incarcerated and nonincarcerated hernia patients were assessed with appropriate statistical tests including the Student's T test or MannWhitney $U$ test for continuous variables and the Fishers' exact test or Chi-square test for categorical variables. To prevent bias, multiple imputations were performed to compensate for missing data. Multiple imputations were performed with five imputations to ensure maximized use of available data. Factors potentially associated with incarceration were assessed in univariate logistic regression. Factors that were potentially related after univariate analysis $(p<0.2)$ and factors of clinical interest were considered for multivariate analysis. Factors with a strong mutual correlation were not fitted simultaneously. Linearity of continuous variables was graphically assessed. A 'full model' containing all variables of interest was reduced, based on the Wald-statistic and backward elimination, to include only those variables that improved discrimination. Defect width was not linearly associated with incarceration and was therefore not fitted as a continuous variable in a separate model. We deviated from the size categories provided by the EHS classification of primary and incisional abdominal wall hernias, since this classification did not provide enough leniency to adequately include small defects in the logistic regression model. Additionally, the EHS classification on defect location was simplified to include less categories to prevent overfitting of the logistic regression models. To prevent overfitting, a maximum of one variable was fitted per approximately ten incarceration events in the final model [14]. Discrimination of the final model was evaluated with the area under the receiver operator characteristic (ROC) curve [15]. A $p$ value of $<0.05$ was considered statistically significant.

\section{Results}

A total of 2352 patients with a primary hernia and 2120 patients with an incisional hernia had data available on incarceration and were subsequently included in this study. In total, $83(3.5 \%)$ of patients with a primary hernia had a non-reducible incarceration, another 106 (4.5\%) had a reducible incarceration. In total, $79(3.7 \%)$ of the patients with an incisional hernia had a non-reducible incarceration, another $93(4.4 \%)$ had a reducible incarceration. The overall proportion of missing data was low: $1.6 \%$ of data was missing throughout the database. The exact number of missing data for each variable is presented in the Supplement. Patient baseline characteristics and hernia characteristics, as well as results after univariate logistic regression, are presented in Tables 1 and 2.

\section{Primary abdominal wall hernia}

Results of univariate analysis are presented in Table 1. In univariate analysis, increasing age, increasing BMI, ASA class III-IV, ascites, and constipation were associated with an incarcerated hernia. Additionally, peri- and infra-umbilical defects were associated with an incarcerated hernia. Compared to defects of 0-1 cm, a defect width of 3-4 cm (OR 3.31, 95\% CI 1.91-5.74), $p<0.0001$ ) was significantly associated with an incarcerated hernia. In fact, of all patients with a defect width of 3-4 cm, 22 of $227(10 \%)$ presented with an incarcerated hernia. In multivariate analysis only age, BMI, sex, constipation, defect width, and 
Table 1 Primary hernias: patient baseline and hernia characteristics

\begin{tabular}{|c|c|c|c|c|}
\hline Variable patient baseline characteristics & $\begin{array}{l}\text { Not incarcerated } \\
N(\%)\end{array}$ & $\begin{array}{l}\text { Incarcerated } \\
N(\%)\end{array}$ & $\begin{array}{l}\text { Odds ratio } \\
\text { OR }(95 \% \mathrm{CI})\end{array}$ & $p$ value \\
\hline Total \# patients & 2269 & 83 & & \\
\hline Age (years)* & $55.4 \pm 14.6$ & $60.0 \pm 17.4$ & $1.02(1.01-1.04)^{* *}$ & 0.0084 \\
\hline BMI $\left(\mathrm{kg} / \mathrm{m}^{2}\right)^{*}$ & $27.8 \pm 6.1$ & $30.3 \pm 7.5$ & $1.06(1.02-1.09)^{* *}$ & 0.0004 \\
\hline Sex $=$ female & $886(39.0)$ & $35(42.2)$ & $1.14(0.73-1.77)$ & 0.57 \\
\hline Current smoking & $514(23.1)$ & $12(15.2)$ & $0.60(0.32-1.12)$ & 0.11 \\
\hline Diabetes mellitus & $136(6.1)$ & $8(9.6)$ & $1.63(0.77-3.46)$ & 0.20 \\
\hline Corticosteroid use & $76(3.4)$ & $4(4.8)$ & $1.38(0.49-3.85)$ & 0.54 \\
\hline Radiotherapy & $19(0.8)$ & $2(2.4)$ & $2.81(0.64-12.26)$ & 0.17 \\
\hline Chemotherapy & $28(1.2)$ & $2(2.4)$ & $1.67(0.38-7.30)$ & 0.49 \\
\hline History of AAA & $6(0.3)$ & $0(0)$ & - & 0.83 \\
\hline Anticoagulant use & $184(8.2)$ & $12(14.5)$ & $1.88(1-3.54)$ & 0.05 \\
\hline History of abdominal wall hernia & 309 (13.7) & $12(14.5)$ & $1.03(0.55-1.92)$ & 0.92 \\
\hline History of inguinal hernia & $213(9.4)$ & $9(10.8)$ & $1.14(0.56-2.30)$ & 0.73 \\
\hline Family history of hernia & $102(4.5)$ & $1(1.2)$ & $0.25(0.03-1.84)$ & 0.17 \\
\hline \multicolumn{5}{|l|}{ ASA classification } \\
\hline I-II & $1912(84.9)$ & $58(70.7)$ & 1 (reference) & \\
\hline III-IV & $340(15.1)$ & $24(29.3)$ & $2.35(1.44-3.83)$ & 0.0006 \\
\hline Ascites & $17(0.8)$ & $3(3.7)$ & $4.48(1.25-16.08)$ & 0.0215 \\
\hline Chronic cough & $107(4.8)$ & $2(2.4)$ & $0.55(0.14-2.18)$ & 0.39 \\
\hline Constipation & $65(2.9)$ & $7(8.5)$ & $3.04(1.34-6.90)$ & 0.0078 \\
\hline Heavy lifting & $225(10.0)$ & $11(13.4)$ & $1.36(0.71-2.61)$ & 0.35 \\
\hline \multicolumn{5}{|l|}{ Hernia characteristics } \\
\hline \multicolumn{5}{|l|}{ Defect location } \\
\hline Supra-umbilical & $526(23.6)$ & $11(13.3)$ & 1 (reference) & \\
\hline Peri- and infra-umbilical & $1659(74.3)$ & $70(84.3)$ & $2.04(1.07-3.89)$ & $\mathbf{0 . 0 3}$ \\
\hline Lateral & $46(2.1)$ & $2(2.4)$ & $1.78(0.37-8.63)$ & 0.48 \\
\hline \multicolumn{5}{|l|}{ Defect width $(\mathrm{cm})$} \\
\hline 1 & $1328(58.9)$ & $40(48.2)$ & 1 (reference) & - \\
\hline 2 & $665(29.5)$ & $19(22.9)$ & $0.95(0.54-1.65)$ & 0.84 \\
\hline $3-4$ & $205(9.1)$ & $22(26.5)$ & $3.31(1.91-5.74)$ & $<0.0001$ \\
\hline$\geq 5$ & $56(2.5)$ & $2(2.4)$ & $1.38(0.32-5.92)$ & 0.66 \\
\hline
\end{tabular}

Statistically significant values $(p<0.05)$ are given in bold

$B M I$ body mass index, $A A A$ abdominal aortic aneurysm, ASA score: American Society of Anesthesiologists score

*Mean \pm SD are presented for age and BMI; **per one increase; $\mathrm{P}$ for Wald-statistic after univariate logistic regression

defect location contributed significantly to discrimination between patients with and without an incarcerated hernia (Table 3). Compared to defects of $0-1 \mathrm{~cm}$, in multivariate analysis, only a defect width of $3-4 \mathrm{~cm}$ (OR $2.85,95 \%$ CI 1.57-5.18, $p=0.0006)$ and peri- and infra-umbilical defects (OR 1.98, 95\% CI 1.02-3.85, $p=0.043$ ) were significantly associated with an incarcerated hernia. In multivariate analysis, ascites and ASA classification were not significantly associated with an incarcerated hernia. The area under the ROC curve for the multivariate model was 0.68 .

\section{Incisional hernia}

Results of univariate analysis are presented in Table 2. In univariate analysis increasing age, increasing BMI, female sex, diabetes mellitus, ASA score III-IV, gynaecologic surgery, and constipation were associated with an incarcerated hernia. No specific defect location (supra-, periand infra-umbilical or lateral) was associated with an incarcerated hernia. Compared to defects of $0-2 \mathrm{~cm}$, a defect width of $3-4 \mathrm{~cm}$ (OR 2.62, 95\% CI 1.32-5.19, $p=0.0057)$ and a defect width $5-10 \mathrm{~cm}$ (OR 2.08, 95\% CI 
Table 2 Incisional hernia: patient baseline and hernia characteristics

\begin{tabular}{|c|c|c|c|c|}
\hline $\begin{array}{l}\text { Variable } \\
\text { Patient baseline characteristics }\end{array}$ & $\begin{array}{l}\text { Not incarcerated } \\
N(\%)\end{array}$ & $\begin{array}{l}\text { Incarcerated } \\
N(\%)\end{array}$ & Odds ratio OR $(95 \% \mathrm{CI})$ & $p$ value \\
\hline Total \# patients & 2041 & 79 & & \\
\hline Age (years)* & $62.7 \pm 14.1$ & $67.9 \pm 13.7$ & $1.03(1.01-1.05)^{* *}$ & 0.0013 \\
\hline $\operatorname{BMI}\left(\mathrm{kg} / \mathrm{m}^{2}\right)^{*}$ & $29.3 \pm 6.1$ & $32.1 \pm 7.9$ & $1.06(1.03-1.09)^{* *}$ & 0.0002 \\
\hline Sex $=$ female & $1050(51.4)$ & $59(74.7)$ & $2.78(1.66-4.66)$ & $<0.0001$ \\
\hline Current smoking & $365(18.8)$ & $10(13.5)$ & $0.73(0.39-1.37)$ & 0.33 \\
\hline Diabetes mellitus & $240(12)$ & $24(31.6)$ & $3.40(2.07-5.57)$ & $<0.0001$ \\
\hline Corticosteroid use & $73(3.6)$ & $2(2.6)$ & $0.71(0.17-2.98)$ & 0.64 \\
\hline Radiotherapy & $36(1.8)$ & $1(1.3)$ & $0.74(0.10-5.16)$ & 0.75 \\
\hline Chemotherapy & $126(6.3)$ & $3(3.9)$ & $0.64(0.20-2.01)$ & 0.44 \\
\hline History of AAA & $15(0.7)$ & $1(1.3)$ & $1.81(0.23-14.35)$ & 0.58 \\
\hline Anticoagulant use & $341(17)$ & $16(21.1)$ & $1.30(0.74-2.29)$ & 0.37 \\
\hline History of abdominal wall hernia & 844 (41.6) & $35(44.9)$ & $1.15(0.73-1.82)$ & 0.54 \\
\hline History of inguinal hernia & $215(10.6)$ & $5(6.4)$ & $0.56(0.22-1.40)$ & 0.21 \\
\hline \multicolumn{5}{|l|}{ ASA classification } \\
\hline $\mathrm{I}-\mathrm{II}$ & $1418(69.7)$ & $33(43.4)$ & 1 (reference) & \\
\hline III-IV & $617(30.3)$ & $43(56.6)$ & $3.04(1.89-4.89)$ & $<0.0001$ \\
\hline \multicolumn{5}{|l|}{ Primary surgery } \\
\hline Gastro-intestinal & $972(48.2)$ & $27(35.5)$ & $0.79(0.45-1.40)$ & 0.42 \\
\hline Gynaecologic & $344(17.1)$ & $25(32.9)$ & $2.12(1.18-3.79)$ & 0.0118 \\
\hline Other & $700(34.7)$ & $24(31.6)$ & 1 (reference) & \\
\hline Ascites & $14(0.7)$ & $0(0)$ & - & 0.85 \\
\hline Chronic cough & $196(9.7)$ & $8(10.4)$ & $1.12(0.54-2.30)$ & 0.76 \\
\hline Constipation & $131(6.5)$ & $11(14.3)$ & $2.33(1.2-4.51)$ & 0.0122 \\
\hline Heavy lifting & $139(6.9)$ & $8(10.4)$ & $1.57(0.74-3.33)$ & 0.07 \\
\hline \multicolumn{5}{|l|}{ Hernia characteristics } \\
\hline \multicolumn{5}{|l|}{ Type of hernia } \\
\hline Recurrent hernia & $410(20.4)$ & $21(28.0)$ & $1.63(0.95-2.77)$ & 0.07 \\
\hline Previous surgery with mesh & $689(34.2)$ & $20(26.7)$ & $0.74(0.44-1.25)$ & 0.26 \\
\hline \multicolumn{5}{|l|}{ Defect location } \\
\hline Supra-umbilical & $359(22.1)$ & $9(15.3)$ & 1 (reference) & \\
\hline Peri- and infra-umbilical & $955(58.7)$ & $45(76.3)$ & $1.80(0.88-3.68)$ & 0.11 \\
\hline Lateral & $288(17.7)$ & $5(8.5)$ & $1.08(0.42-2.81)$ & 0.87 \\
\hline \multicolumn{5}{|l|}{ Defect width $(\mathrm{cm})$} \\
\hline $0-2$ & 567 (28.6) & $11(14.7)$ & 1 (reference) & - \\
\hline $3-4$ & $632(31.9)$ & $34(45.3)$ & $2.62(1.32-5.19)$ & 0.0057 \\
\hline $5-10$ & $658(33.2)$ & $27(36.0)$ & $2.08(1.02-4.27)$ & 0.0450 \\
\hline$>10$ & $124(6.3)$ & $3(4.0)$ & $1.32(0.39-4.51)$ & 0.66 \\
\hline
\end{tabular}

Statistically significant values $(p<0.05)$ are given in bold

$B M I$ body mass index, $A A A$ abdominal aortic aneurysm, ASA score American Society of Anesthesiologists score

*Mean \pm SD are presented for age and BMI; **per one increase; $\mathrm{P}$ for Wald-statistic after univariate logistic regression

$1.02-4.27, p=0.045$ ) were significantly associated with an incarcerated hernia. In multivariate analysis, only age, BMI, sex, diabetes mellitus, heavy lifting, ASA classification, and defect width contributed significantly to discrimination between patients that presented with and without an incarcerated hernia (Table 3). Compared to defects of $0-2 \mathrm{~cm}$, in multivariate analysis, only a defect width of 3-4 cm was significantly associated with an incarcerated hernia (OR 2.14, 95\% CI 1.07-4.31, $p=0.0324)$. In multivariate analysis, gynaecologic surgery, constipation, and defect location were not significantly associated with patients that presented with an incarcerated hernia. The area under the ROC curve for the multivariate model was 0.76 . 
Table 3 Results of multivariate logistic regression

\begin{tabular}{|c|c|c|c|}
\hline & Coefficient & Odds ratio $(95 \% \mathrm{CI})$ & $p$ value \\
\hline \multicolumn{4}{|l|}{ Primary hernias } \\
\hline Intercept & -6.1051 & - & - \\
\hline $\begin{array}{l}\text { Age (years), per one } \\
\text { increase }\end{array}$ & 0.0167 & $1.02(1-1.03)$ & 0.0421 \\
\hline $\begin{array}{l}\mathrm{BMI}\left(\mathrm{kg} / \mathrm{m}^{2}\right) \text {, per one } \\
\text { increase }\end{array}$ & 0.0341 & $1.03(1-1.07)$ & $\mathbf{0 . 0 3 7 7}$ \\
\hline Sex $=$ female & 0.2767 & $1.32(0.83-2.09)$ & 0.24 \\
\hline Constipation & 0.934 & $2.54(1.08-6.02)$ & $\mathbf{0 . 0 3 3 5}$ \\
\hline \multicolumn{4}{|l|}{ Defect location } \\
\hline Supra-umbilical & Reference & 1 (reference) & \\
\hline Peri- and infra-umbilical & 0.6844 & $1.98(1.02-3.85)$ & 0.043 \\
\hline Lateral & 0.1506 & $1.16(0.24-5.69)$ & 0.85 \\
\hline \multicolumn{4}{|l|}{ Defect width $(\mathrm{cm})$} \\
\hline 1 & Reference & 1 (reference) & \\
\hline 2 & -0.1703 & $0.84(0.48-1.49)$ & 0.56 \\
\hline $3-4$ & 1.0488 & $2.85(1.57-5.18)$ & 0.0006 \\
\hline$\geq 5$ & 0.0637 & $1.07(0.24-4.83)$ & 0.93 \\
\hline \multicolumn{4}{|l|}{ Incisional hernias } \\
\hline Intercept & -8.5286 & - & - \\
\hline $\begin{array}{l}\text { Age (years), per one } \\
\text { increase }\end{array}$ & 0.0251 & $1.03(1.01-1.05)$ & 0.0122 \\
\hline $\begin{array}{l}\mathrm{BMI}\left(\mathrm{kg} / \mathrm{m}^{2}\right), \text { per one } \\
\text { increase }\end{array}$ & 0.0342 & $1.03(1-1.07)$ & 0.06 \\
\hline Sex $=$ female & 1.0431 & $2.84(1.66-4.87)$ & 0.0001 \\
\hline Diabetes mellitus & 0.8384 & $2.31(1.37-3.91)$ & 0.0017 \\
\hline Heavy lifting & 0.9882 & $2.69(1.17-6.16)$ & 0.0196 \\
\hline \multicolumn{4}{|l|}{ ASA classification } \\
\hline I-II & Reference & 1 (reference) & \\
\hline III-IV & 0.8124 & $2.25(1.34-3.78)$ & 0.0021 \\
\hline \multicolumn{4}{|l|}{ Defect width $(\mathrm{cm})$} \\
\hline $0-2$ & Reference & 1 (reference) & \\
\hline $3-4$ & 0.7627 & $2.14(1.07-4.31)$ & 0.0324 \\
\hline $5-10$ & 0.569 & $1.77(0.84-3.7)$ & 0.13 \\
\hline$>10$ & 0.1598 & $1.17(0.33-4.15)$ & 0.80 \\
\hline
\end{tabular}

Statistically significant values $(p<0.05)$ are given in bold

$B M I$ body mass index, ASA score American Society of Anesthesiologists score; P for Wald-statistic after multivariate logistic regression

\section{Discussion}

In this large prospective study within the French HerniaClub registry of patients with primary and incisional hernias, a number of factors were associated with patients that had presented with either an incarcerated primary or incisional hernia. For both primary and incisional hernias, a defect width of 3-4 cm portrayed the highest odds (OR 2.85 and OR 2.14, respectively) for an incarcerated hernia. Probably defects $\leq 2 \mathrm{~cm}$ in width would still be too small to facilitate substantial protrusion of abdominal contents in most cases, whereas larger hernias would be too large to cause for substantial strangulation. For primary hernias, periumbilical and umbilical defects were associated with an increased odds for incarceration (OR 1.98), defect location was not associated with incarceration for incisional hernias.

Findings of a previous prospective cohort study assessing factors associated with emergency surgery in patients with abdominal wall hernias are reasonably similar to the present results, finding female sex and age to be associated with emergency surgery [6]. In this same study, the relation between defect size and emergency surgery in incisional hernias was disconcordant with the present results. This is 
likely due to different size categories used in this report; all defects between 2 and $7 \mathrm{~cm}$ were grouped together. Another retrospective study evaluated hernia characteristics as risk factors for incarceration in patients with a primary or incisional hernia [4]. In contrast to the present results, this study did not report a significant association between defect size and incarceration. However, in this study, patients with a primary and incisional hernia were grouped together. Nonetheless, the aetiology of both conditions is likely to be different [16]. This discrepancy could likewise be caused due to the fact that defect width was fitted as a continuous variable in the multivariate logistic regression model, whereas, in the present analysis, this relation was not linear. The authors additionally found hernia sac height and angle between the hernia sac and abdominal wall (on CT-scan) to be associated with incarceration.

The present study additionally found numerous patient factors to be associated with incarceration for either primary or incisional hernias. Increased BMI was correlated with incarceration. Additionally, it is conceivable that factors increasing abdominal pressure including constipation and heavy lifting may be associated with incarceration. Other variables found to be associated, including age and ASA classification, may be secondary effects to variables which are not available in this current database. For example, clinicians may be more reluctant to operate older patients with higher ASA classification, resulting in increased odds of these patients being operated in an emergency setting due to incarceration. Nevertheless, it is conceivable that frail patients are at increased odds for a complicated prognosis. Patients with an incisional hernia, female sex was associated with incarceration (OR 2.31); however, in primary hernias, this association was not present. The reason for this association remains unclear. Although previous studies failed to show a strong correlation between pregnancy and hernia occurrence, the increased odds for incarceration in women may be related to physiological changes in the abdominal wall secondary to pregnancy $[17,18]$.

A strangulated and non-reducible hernia is an absolute indication for emergency surgery and causes for increased morbidity and mortality $[2,3,5,6]$. Patients with incarcerated hernias are hospitalized longer and suffer from increased rates of severe postoperative complications [2-4]. Moreover, rates of emergency hernia repair have been increasing in the USA over the past years [19]. This might be related to an overall increase in prevalence of abdominal hernias [4]. Therefore, data constituting the prevention of incarceration is important and may improve clinical care and decision making.

The present and previous reports suggest that incarceration is, to a certain extent, predictable based on patient factors, hernia characteristics, and CT-findings. Nevertheless, in order to better predict which patients may be at increased risk for incarceration, future prospective cohorts require inclusion of those patients treated conservatively, CT-scans for additional biometric evaluation, and inclusion of time to event data. This would ensure accurate depiction of the complete order of events.

\section{Limitations}

Although all data was collected prospectively in an exhaustive manner, results may be influenced by selection bias to a certain degree, given the observational study design. All included patients underwent hernia repair surgery. Patients who were treated conservatively were not included in this registry. Therefore, causality of found associations cannot be confirmed. Additionally, this limits the current potential to make accurate probability estimates. Patients presenting in an emergency setting may not be operated by a dedicated hernia surgeon affiliated with the Hernia-Club registry and may be less likely to be included in the registry database. However, this will likely have non-differential effects on reported odds ratios. Nevertheless, this may cause for an underestimation of the reported prevalence of incarceration. The proportion of missing data was reasonably low and multiple imputations were used to ensure maximized use of available data. Inherently, it was not possible to provide exact reasons for missing data at case and variable level. Therefore, a risk of reporting bias cannot be completely excluded. To allow for better interpretation and adequate effect estimation, defect width was categorized. However, in reality, no strict cutoffs exist and these estimates will merely represent an approximation of the true effects.

\section{Conclusion}

For primary and incisional hernias, mainly defects of 3-4 cm were associated with incarceration. For primary hernias, mainly defects located in the peri- and infra-umbilical region were associated with incarceration. Probably defects of $\leq 2 \mathrm{~cm}$ in width would still be too small for substantial protrusion of abdominal contents in most cases, whereas larger hernias would be too large to cause for strangulation. Based on patient and hernia characteristics, patients with increased odds for incarceration may be selected and these patients may benefit from elective surgical treatment.

Acknowledgements Members of the Hernia-Club: Antor M (CHRU Rouen, Rouen, France), Beck M (Clinique Ambroise Paré, Thionville, France), Barrat C (Hôpital Universitaire Jean Verdier, Bondy, France), Berney C (Bankstown-Lidcombe Hospital, Sydney, Australia), Binot D (MCO Côte d'Opale, Boulogne sur Mer, France), 
Bousquet J (Hôpital Privé de la Chataigneraie, Montpellier, France), Blazquez D (Clinique Jeanne d'Arc, Paris, France), Bonan A (Hôpital Privé d'Antony, Antony, France), Cas O (Centre Médico ChirurgicalFondation Wallerstein, Arès, France), Champault-Fezais A (Groupe Hospitalier Paris St Joseph, Paris, France), Chastan P (Bordeaux, France), Chollet J-M (Hôpital Privé d'Antony, Antony, France), Cossa J-P (CMC Bizet, Paris, France), Dabrowski A (Clinique de Saint Omer, Saint Omer, France), Delaunay T (Clinique St Hilaire, Rouen, France), Démaret $\mathrm{S}$ (Clinique Saint Vincent, Besançon, France), Drissi F (CHU Nantes, Nantes, France), Demian H (CHU Croix Rousse, Lyon, France), Dubuisson V (CHU Pellegrin, Bordeaux, France), Dugue $\mathrm{T}$ (Clinique de Saint Omer, Saint Omer, France), Fromont G (Clinique de Bois Bernard, Bois Bernard, France), Gillion J-F (Hôpital Privé d'Antony, Antony, France), Jacquin $\mathrm{C}(\mathrm{CH}$ du Prado, Marseille, France), Jurczak F (Clinique Mutualiste, Saint Nazaire, France), Khalil H (CHRU Rouen, Rouen, France), Launay-Savary M (CHU Pellegrin, Bordeaux, France), Lepère M (Clinique Saint Charles, La Roche-sur-Yon, France), Lépront D (Polyclinique de Navarre, Pau, France), Longeville JH (Clinique du Val-de-Loire, Nevers, France), Le Toux N (Clinique Jeanne d'Arc, Paris, France), Loriau J (Groupe Hospitalier Paris St Joseph, Paris, France), Magne E (Clinique Tivoli, Bordeaux, France), Ngo P (Hôpital Américain, Neuilly, France), Oberlin O (Croix St Simon Diaconesses, Paris, France), Passot G (CHU de Lyon sud, Lyon, France), Pavis d'Escurac X (Strasbourg, France), Putinier JB (CH mutualiste, grenoble, France), Renard Y (CHRU Reims, Reims, France), Romain B (CHU Strasbourg, Strasbourg, France), Soler M (Polyclinique Saint Jean, Cagnes-sur-Mer, France), Roos S (Clinique Claude Bernard, Albi, France), Thillois J-M (Hôpital Privé d'Antony, Antony, France), Tiry P (Clinique de Saint Omer, Saint Omer, France), Vu P (HPMV, Bry sur Marne, France), Verhaeghe R (MCO Côte d'Opale, Boulogne sur Mer, France), Warlaumont M (CHRU Lille, Lille, France) and Zaranis C (Clinique de La Rochelle, France).

\section{Compliance with ethical standards}

Conflict of interest The authors declare no conflict of interest related to the submitted work.

Open Access This article is distributed under the terms of the Creative Commons Attribution 4.0 International License (http://crea tivecommons.org/licenses/by/4.0/), which permits unrestricted use, distribution, and reproduction in any medium, provided you give appropriate credit to the original author(s) and the source, provide a link to the Creative Commons license, and indicate if changes were made.

\section{References}

1. van Ramshorst GH et al (2012) Impact of incisional hernia on health-related quality of life and body image: a prospective cohort study. Am J Surg 204(2):144-150
2. Li LT et al (2014) Outcomes of acute versus elective primary ventral hernia repair. J Trauma Acute Care Surg 76(2):523-528

3. Chung PJ et al (2017) Predicting 30-day postoperative mortality for emergent anterior abdominal wall hernia repairs using the American College of Surgeons National Surgical Quality Improvement Program database. Hernia 21(3):323-333

4. Mueck KM et al (2017) Computed tomography findings associated with the risk for emergency ventral hernia repair. Am J Surg 214(1):42-46

5. Jensen KK, Erichsen R, Krarup PM (2017) The impact of incisional hernia on mortality after colonic cancer resection. Surg Endosc 31(5):2149-2154

6. Helgstrand F et al (2013) Outcomes after emergency versus elective ventral hernia repair: a prospective nationwide study. World J Surg 37(10):2273-2279. https://doi.org/10.1007/s00268013-2123-5

7. Itatsu $\mathrm{K}$ et al (2014) Incidence of and risk factors for incisional hernia after abdominal surgery. Br J Surg 101(11):1439-1447

8. Lau B et al (2012) Obesity increases the odds of acquiring and incarcerating noninguinal abdominal wall hernias. Am Surg 78(10):1118-1121

9. Marsman HA et al (2007) Management in patients with liver cirrhosis and an umbilical hernia. Surgery 142(3):372-375

10. Vandenbroucke JP et al (2007) Strengthening the Reporting of Observational Studies in Epidemiology (STROBE): explanation and elaboration. PLoS Med 4(10):e297

11. Muysoms F et al (2012) EuraHS: the development of an international online platform for registration and outcome measurement of ventral abdominal wall hernia repair. Hernia 16(3):239-250

12. Muysoms FE et al (2009) Classification of primary and incisional abdominal wall hernias. Hernia 13(4):407-414

13. van Buuren S, Groothuis-Oudshoorn K (2010) mice: multivariate imputation by chained equations in R. J Stat Softw 45:1-68

14. Steyerberg EW, Eijkemans MJ, Habbema JDF (1999) Stepwise selection in small data sets: a simulation study of bias in logistic regression analysis. J Clin Epidemiol 52(10):935-942

15. Hanley JA, McNeil BJ (1982) The meaning and use of the area under a receiver operating characteristic (ROC) curve. Radiology 143(1):29-36

16. Kroese LF et al (2018) Primary and incisional ventral hernias are different in terms of patient characteristics and postoperative complications-a prospective cohort study of 4,565 patients. Int J Surg 51:114-119

17. Boissonnault JS, Blaschak MJ (1988) Incidence of diastasis recti abdominis during the childbearing year. Phys Ther 68(7):1082-1086

18. Akram J, Matzen SH (2014) Rectus abdominis diastasis. J Plast Surg Hand Surg 48(3):163-169

19. Beadles CA, Meagher AD, Charles AG (2015) Trends in emergent hernia repair in the United States. JAMA Surg 150(3):194-200

Publisher's Note Springer Nature remains neutral with regard to jurisdictional claims in published maps and institutional affiliations. 